\title{
Cone beam computed tomography after round window vibroplasty: do the radiological findings match the auditory outcome?
}

\section{Maurizio Barbara, Luigi Volpini, Mario Ciotti, Chiara Filippi, Edoardo Covelli, Simonetta Monini \& Ferdinando D’Ambrosio}

To cite this article: Maurizio Barbara, Luigi Volpini, Mario Ciotti, Chiara Filippi, Edoardo Covelli, Simonetta Monini \& Ferdinando D'Ambrosio (2015) Cone beam computed tomography after round window vibroplasty: do the radiological findings match the auditory outcome?, Acta OtoLaryngologica, 135:4, 369-375

To link to this article: http://dx.doi.org/10.3109/00016489.2014.990582

Published online: 06 Mar 2015.

Submit your article to this journal

Џ Article views: 35

Q View related articles ๘

View Crossmark data $₫$ 


\title{
Cone beam computed tomography after round window vibroplasty: do the radiological findings match the auditory outcome?
}

\author{
MAURIZIO BARBARA ${ }^{1}$, LUIGI VOLPINI ${ }^{1}$, MARIO CIOTTI $^{2}$, CHIARA FILIPPI ${ }^{1}$, \\ EDOARDO COVELLI ${ }^{1}$, SIMONETTA MONINI ${ }^{1}$ \& FERDINANDO D'AMBROSIO ${ }^{2}$
}

\author{
${ }^{1}$ ENT Unit, NESMOS Department, Medicine and Psychology and Medicine, Sapienza University, Rome, Italy and \\ ${ }^{2}$ Radiology Department, Odontoiatrics and Medicine, Sapienza University, Rome, Italy
}

\begin{abstract}
Conclusion: The cone beam computed tomography (CBCT) imaging technique has proved to be reliable for assessing the appropriate positioning of the floating mass transducer (FMT) in the round window (RW) niche, although some parameters do not seem to be essential for achieving a satisfactory functional outcome. Objectives: To evaluate the role that specific imaging parameters derived from CBCT of the temporal bone have for predicting the functional outcome after RW vibroplasty (RWVP). Methods: CBCT imaging was carried out in a homogeneous group of patients who presented with a mixed type of hearing loss after open tympanoplasty. Three arbitrary radiological parameters were taken into account: the FMT/RW membrane contact, bony contacts of the FMT margins, and the inferior FMT tissue support. The audiological assessment took into consideration the PTA4 (500-4000 Hz), the PTA2 $(125-250 \mathrm{~Hz})$, and the word recognition score (WRS) in quiet and in noise. Results: One subject presented with all positive CBCT parameters and showed a good, but not the best auditory performance among the study group. In the majority of the subjects, with a satisfactory postoperative hearing improvement, at least two of the three radiological parameters were present. In comparison with the unaided condition, an improvement in both the PTA4 and PTA2 was found in all the subjects.
\end{abstract}

Keywords: mixed hearing loss, pure-tone average, word recognition score

\section{Introduction}

Active middle ear implants (AMEIs), first introduced for the rehabilitation of sensorineural hearing loss, have successively also been indicated in selected cases with conductive or mixed type of hearing loss [1]. Since then, AMEIs have been proposed for improving hearing in clinical situations that contraindicate or make unfeasible the adoption of conventional airconductive hearing aids (HAs), such as in presence of ear malformations, multiple unsuccessful ossicular reconstructions or sequels from specific surgical procedures [2-4]. In the latter case, it is possible to individuate a clinical category represented by those patients who have undergone an open tympanoplasty procedure, formerly known as radical mastoidectomy, in whom the inner ear function may show deterioration - such as bone conduction (BC) threshold - and the traditional reconstruction techniques with passive ossicular prostheses (PORP, TORP) may be either insufficient to provide a useful hearing or, rather, likely to fail, especially when the stapes superstructure is missing. This observation, along with the impossibility of wearing a conventional air-conductive HA or a BC implant if the BC threshold exceeds the 40$45 \mathrm{~dB}$ limit, has opened the door for the adoption of an AMEI to be placed on the ossicular remnants, such as the stapes or the footplate $[5,6]$, or the round window (RW) region, in close contact with the RW membrane (RWM) [1]. Previous clinical reports have shown that RWM-coupled AMEIs could produce good functional results, thus indicating them to be

Correspondence: Maurizio Barbara, Chairman of Otorhinolaryngology, University Hospital Sant'Andrea, Sapienza, Via Di Grottarossa 1035, 00189 Rome, Italy Tel: +3906 33775628; Fax: +3906 33775058; E-mail: maurizio.barbara@uniroma1.it 
reliable tools for hearing rehabilitation after anatomic sequels from middle ear surgery [2-7]. Earlier, in 1948, this method of inner ear stimulation was described in detail by Wever and Lawrence [8], who were able to prove that, via the RWM, a cochlear stimulation nearly identical to the classic oval window pathway can be achieved.

The present study was designed to evaluate if the functional outcome after the surgical coupling of an AMEI on the RWM corresponds to its optimal surgical positioning, as radiologically assessed by a cone beam computed tomography (CBCT) study, performed at a certain distance from the surgical implantation.

\section{Material and methods}

Eight of 14 subjects who underwent the surgical application of an AMEI for a mixed type of hearing loss were included in this study, while the other 6 were excluded for different reasons, such as deceased (1 case), follow-up was too short (2 cases), sudden deafness 20 days after surgery ( 1 case), oval window coupling (1 case), and inappropriate indication (phantom preoperative $\mathrm{BC}$ threshold, 1 case). In the past (i.e. 5-15 years earlier) all the subjects had undergone an open tympanoplasty procedure for cholesteatoma, with a rather stable local condition only needing periodic cleansing from debris and wax. Due to a mild impairment of the contralateral ear, five subjects had previously experienced wearing of a glass-coupled bone-conducting HA, which over time was shown to provide an insufficient functional gain and prompted them to ask for an alternative rehabilitative solution at our Implanting Centre. The demographics of the subjects are presented in Table I. As routinely performed at our Implanting Centre in case of conductive/mixed hearing loss, also in view of the implantation of a middle ear device [9], all the subjects underwent a full set of auditory tests with the headband coupled to a high-power BC device, which included soundfield pure-tone and speech audiometry, in quiet and in noise, with the contralateral ear plugged. The Vibrant Soundbridge ${ }^{\circledR}$ or VSB (Medel, Innsbruck, Austria) was selected as the AMEI for this study. The RW vibroplasty (RW-VP) procedure was performed by the same surgeon under general anesthesia, with the following standardized steps: retroauricular incision; en bloc de-epithelialization of the cavity until complete exposure of the medial wall of the middle ear cavity was achieved; identification of the RW niche; drill-out of its bony borders, aiming to achieve the maximal exposure of the RWM; positioning of the internal receiver and connector link and placement of the floating mass transducer (FMT), with particular care in placing the upper side of the FMT in contact with the RWM, with fascia interposition in three cases; placement of supporting tissue, made of small cartilage pieces, inferiorly to the FMT, to provide stabilization and tension of the FMT itself. This assemblage was further covered by fascia and cartilage and, finally, the epithelial lining was repositioned, repairing eventual defects with an underlay placement of heterologous fascia tissue (Tutopatch ${ }^{\circledR}$, MED \& CARE, Gdynia, Poland).

Occasionally, an objective procedure was performed after FMT placement to validate its appropriate inner ear coupling: electrocochleography in five cases [10], via a Labat Audiological Platform (Mogliano Veneto, Italy), by placing a needle electrode on the promontory wall, anterior to the FMT implantation site; the recently implemented device available from the company (VSB QuickCheck ${ }^{\circledR} \mathrm{u}$, Medel, Innsbruck, Austria) - in the last three cases - to rapidly test the proper functioning of the VSB implant, for immediate confirmation of implant integrity.

The mean surgical time was $140 \mathrm{~min}$ (range 110-180 $\mathrm{min}$ ). The patients were discharged from the hospital the day after surgery and the implant was

Table I. Demographics of patients in the study group who underwent round window vibroplasty (RW-VP).

\begin{tabular}{lllll}
\hline Patient no. & Age (years) & Gender & Time (years) from CHO surgery & Time from RW-VP to CTCB \\
\hline 1 & 65 & F & 2 & 2 years, 10 months \\
2 & 41 & F & 4 & 2 years, 4 months \\
3 & 74 & F & 6 & 3 years, 9 months \\
4 & 53 & F & 2 & 5 years, 1 month \\
5 & 41 & F & 3 & 3 years, 2 months \\
6 & 47 & F & 10 & 4 years, 3 months \\
7 & 56 & M & 3 & 1 year, 9 months \\
8 & 60 & F & 23 & 1 year, 10 months \\
\hline
\end{tabular}

CHO: Cholesteatoma; CTCB: Cone beam computed tomography. 
activated 4-6 weeks after surgery. The first complete postoperative audiological assessment, using the same battery of tests as carried out preoperatively, was then performed 6 months after the activation and at the time of the present evaluation ( $\min 21, \max 61$ months; average 3 years and 2 months). In conjunction with the last audiological assessment, all the subjects underwent CBCT (NEWTOM 5G, QR, Verona, Italy) of the temporal bone. A high-frequency $\mathrm{X}$-ray source with rotating anode was used, with a focal spot of $0.3 \mathrm{~mm}$. The field of view was $88 \mathrm{~cm}$, with a high-resolution scan time of $36 \mathrm{~s}$, X-ray emission time of $6,7 \mathrm{~s}, \mathrm{~mA} 1-20$ (pulsed mode), and $\mathrm{kV}$ $110 \mathrm{n}$. The patients lay in a supine position during the examination. After the acquisition, a Class II Medical Device for diagnostic imaging in medicine that fully supports DICOM (Digital Imaging and COmmunications in Medicine) data processing (OsiriX ${ }^{\circledR}$, Geneva, Switzerland), was used for 3D multiplanar reconstructions (MPRs). With a modified Stenvers plane, the basal turn of the cochlea was first identified and images perpendicular to this plane were obtained with the coronal reconstruction (Figure 1a,b). According to this procedure, a similar CT image could be retrieved for all the patients, which included (in a single slice) all the cochlear turns, the vestibule at the stem of the superior and lateral semicircular canal, and the RW niche. A direct visualization of the FMT within the middle ear cavity, with its relationships with the nearby anatomic structures was therefore possible [11].

\section{Evaluation of the images}

Three independent observers evaluated the CBCT images: one neuroradiologist with more than 30 years experience in ear imaging and two ENT surgeons (one 4th year resident; one staff with over 30 years of

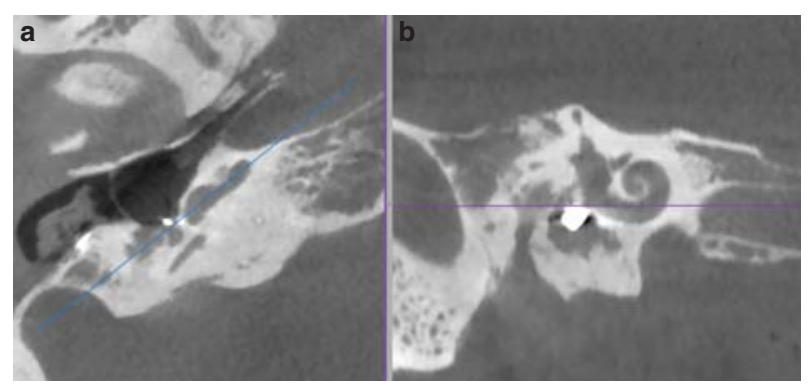

Figure 1. Reconstruction technique for cone beam computed tomography (CBCT) of the temporal bone. (a) After processing the DICOM files with Osirix for Mac, a 3D reconstruction was obtained moving the section in the axial plane so that it runs parallel to the basal turn of the cochlea (Stenvers' modified plane). (b) The coronal reconstruction of this plane allows visualization of the entire cochlear turns, the semicircular canals, and the floating mass transducer (FMT) in the round window niche in the same section. ear surgery and 5 years experience in VSB surgery). All the observers were familiar with the operating technique itself but were not provided with any information on patient data. Every observer had to assess the proposed variant of coupling on separate sheets, obtaining three different sheets for each patient. Each observer independently visualized the FMT device. The standardized view was set by the neuroradiologist, who furnished his evaluation, and then let the other two observers sit at the workstation and provide an independent judgment on the position of the FMT within the middle ear cavity. Three CT parameters were arbitrarily taken into consideration for this purpose, as follows. i) The contact of the upper side of the FMT with the RWM; ii) the eventual presence of bony contacts; iii) the contact and continuity of the lower side of the FMT with the supporting tissue.

Accordingly, for all the subjects, it was possible to rate the CT item as poor $(+)$, good $(++)$, or optimal $(+++)$ contact (parameter no. 1) (Figure 2a, b, c); and as 'Yes' or 'No' for parameter nos 2 and 3. The evaluation sheet for each single CT exam was numbered, without any further note for the identification of the examined patient. Moreover, neither of the two ENT observers was aware of the audiological data of the patients that were obtained and collected by an audiologist at the same clinic, and progressively numbered as well. For the audiological assessment, the following data were retrieved: the pure-tone average for frequencies between 500 and $4000 \mathrm{~Hz}$ (PTA4); the pure-tone average for 125 and $250 \mathrm{~Hz}$; the soundfield speech reception threshold as $50 \%$ of sound delivered at $65 \mathrm{~dB}$ in quiet and in noise $(\mathrm{S} / \mathrm{N}$ $\mathrm{R}=+10$ ); the sound-field percentage of word discrimination score (WRS) in quiet and noise. The audiological results were graded as optimal when the PTA4 gain was greater than $40 \mathrm{~dB}$, with WRS $>40 \%$ in quiet and $40 \%$ in noise; good if the PTA4 gain was within 20-40 dB, with WRS $20-39 \%$ in quiet and $20-39 \%$ in noise; and poor if the PTA4 gain was between 0 and $20 \mathrm{~dB}$, with WRS $0-19 \%$ in quiet and $0-19 \%$ in noise. Comparison between the unaided and the post-RW-VP condition was performed.
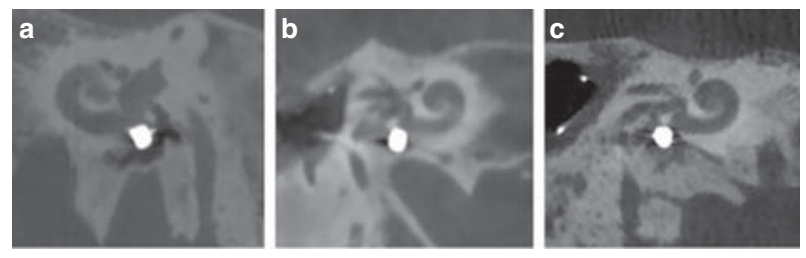

Figure 2. The reconstructed images show examples in reference to the main parameter taken into consideration, i.e. the floating mass transducer/round window membrane (FMT)/RWM contact, as optimal (a), good (b), or poor (c). 
The present study was performed in accordance with the ethical standards of the committee on human experimentation of Sapienza University and with the Helsinki Declaration of 1975, as revised in 1983.

\section{Results}

The surgical procedure was completed without any complication in all the subjects. The bony rim of the RW niche was drilled via a 0.5 diamond bur, more extensively in the region of the superior lip, until the complete superior annular attachment of the RWM was visualized. The drilling was also performed in the medial promontory wall, inferiorly to the RW niche, to accommodate the lateral, convex side of the FMT. In six of the eight subjects, the RW niche was occupied by fibrous tissue that was totally removed by micro-hooks and sickle knife in three of them. In these three subjects, the FMT was placed with fascia interposition (perichondrium from the tragal cartilage). In the remaining three subjects, debridement of the fibrous tissue was performed in close proximity to the RWM, until the 'window play' effect when touching the stapes footplate was visualized. In these subjects, the FMT was directly located into the RW niche without fascia interposition. After its placement, the FMT was supported inferiorly by small chips of tragal cartilage, placed as a palisade at the hypotympanic level. After stabilization of the device, intraoperative electrocochleography was attempted in five of the eight subjects, being successful in only three of them (Figure 3). After activation of the device, which occurred 4-6 weeks after surgery, the functional gain was evaluated by setting the amplification parameters to a comfortable level of loudness. At 3 months after surgery, further adjustments were performed to get a final setting for each patient. At this time the audiological assessment was performed. Data relative to the final evaluation that was performed during the same period at different times from surgery, were considered as definitive and taken into consideration for this study (Table I). Pure-tone and speech audiometry results are shown in Table II. According to the grading system for the audiological evaluation, results for five patients were rated as optimal, two as good, and two as poor. According to the radiological patterns, one subject was rated as optimal (optimal FMT/RWM contact plus no bony contact and good inferior support), six as good, and two as poor. After collecting these individual values, the data were cross-matched to see if there was evidence that the audiological outcome was correlated with the FMT placement. The patient (no. 3) who showed the best radiological score did not achieve the best auditory outcome. In the three subjects (nos 1, 4, and 5) who showed the optimal auditory outcome, both positive and negative imaging parametric factors were present. Only three subjects (nos 3, 6, and 9) showed poor audiological results but none of them showed totally negative imaging parameters.

\section{Discussion}

In respect to the past, in which the principles of chronic ear surgery only addressed the recovery of a safe anatomic condition via obliteration of the open cavity with different modalities (muscle, bone, cartilage) and the use of passive middle ear implants, such

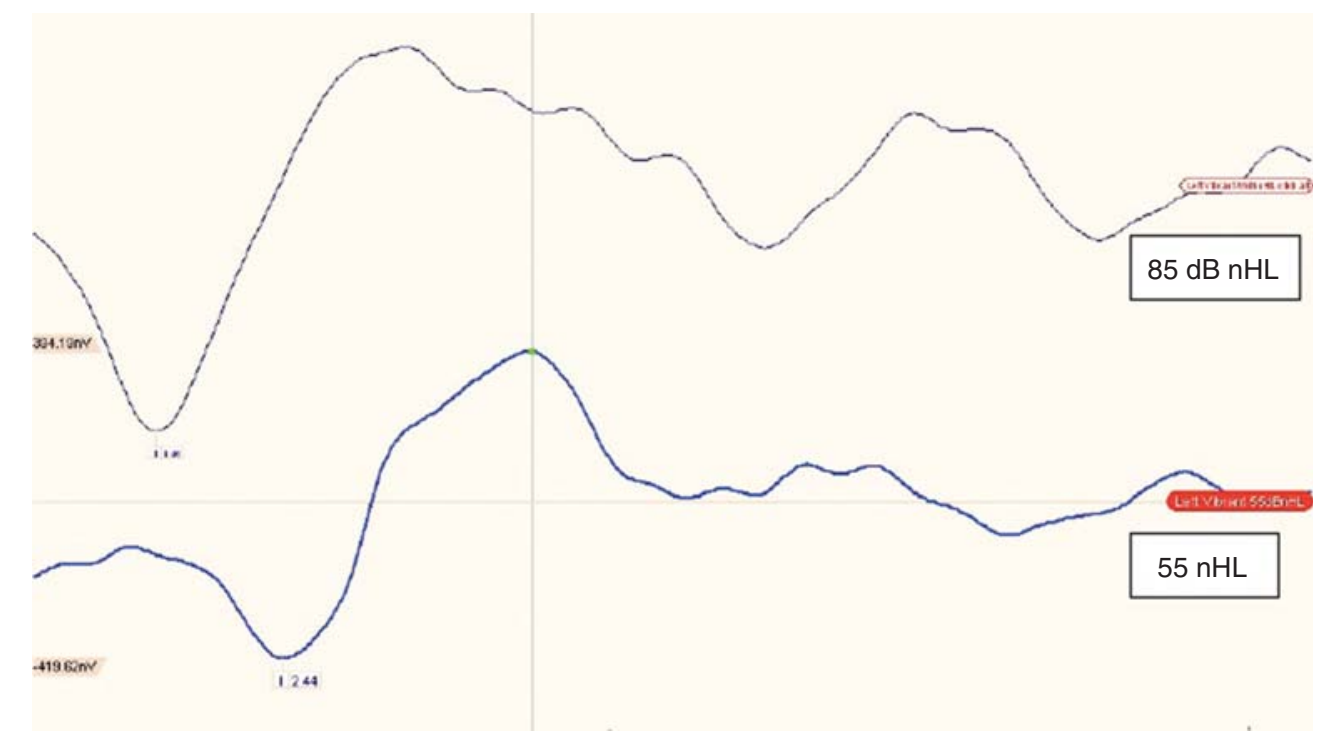

Figure 3. The electrocochleographic trace obtained intraoperatively in patient no. 5, soon after the placement and stabilization of the floating mass transducer (FMT). A clear action potential is shown with changes in amplitude and latency according to the sound stimulation level. 
Table II. Functional and radiological results in the individual cases that underwent round window vibroplasty (RW-VP).

\begin{tabular}{|c|c|c|c|c|c|c|c|c|c|c|c|c|}
\hline \multirow[b]{2}{*}{ Patient no. } & \multicolumn{3}{|c|}{ SF PTA4 $(0.5-4 \mathrm{kHz})$} & \multicolumn{3}{|c|}{ SF WRS in quiet $(\% / \mathrm{dB})$} & \multicolumn{3}{|c|}{$\begin{array}{c}\text { SF WRS in noise } \\
(\mathrm{SNR}=+10)(\% / \mathrm{dB})\end{array}$} & \multicolumn{3}{|c|}{ CTCB parameters } \\
\hline & $\mathrm{U}$ & RW-VP & $\Delta$ & $\mathrm{U}$ & RW-VP & $\Delta$ & $\mathrm{U}$ & RW-VP & $\Delta$ & FMT/RWM contact & $\mathrm{BC}$ & IS \\
\hline 1 & 96 & 47 & +49 & $60 / 80$ & $100 / 60$ & $+40 /-20$ & $70 / 80$ & $70 / 60$ & $0 /-20$ & ++ & Yes & No \\
\hline 2 & 76 & 68 & +8 & $80 / 80$ & $100 / 70$ & $+20 /-10$ & $50 / 80$ & $60 / 70$ & $+10 /-10$ & ++ & Yes & Yes \\
\hline 3 & 98 & 85 & +13 & $10 / 80$ & $80 / 80$ & $+70 / 0$ & 0 & $60 / 80$ & $+60 / 0$ & +++ & No & No \\
\hline 4 & 102 & 52 & +50 & $0(80$ & $100 / 75$ & $+100 /-5$ & 0 & $30 / 75$ & $+30 /-5$ & +++ & Yes & Yes \\
\hline 5 & 108 & 62 & +46 & $40 / 80$ & $100 / 60$ & $+60 /-20$ & 0 & $80 / 60$ & $+80 /-20$ & ++ & Yes & No \\
\hline 6 & 82 & 86 & -4 & $60 / 80$ & $90 / 80$ & $+30 / 0$ & $30 / 80$ & $30 / 80$ & $0 / 0$ & ++ & Yes & Yes \\
\hline 7 & 86 & 63 & +23 & $50 / 80$ & $100 / 70$ & $+50 /-10$ & 0 & $50 / 70$ & $+50 /-10$ & ++ & Yes & No \\
\hline 8 & 70 & 37 & +33 & $100 / 70$ & $100 / 40$ & $0 /-30$ & $90 / 70$ & $70 / 40$ & $-20 /+30$ & +++ & Yes & Yes \\
\hline
\end{tabular}

$\Delta$ : Difference value; BC: Bony contact; CTCB: Cone beam computed tomography; FMT: Floating mass transducer; IS: Inferior support; PTA4: Pure-tone average in the 500-4000 Hz frequency range; RWM: Round window membrane; SF: Sound field; U: Unaided; WRS: Word recognition score.

as PORPs or TORPs, the advent of implantable devices has certainly contributed to modification of the otosurgeon's attitude and has widened the functional possibilities. Nowadays, in fact, different implantable options are available on the basis of the severity of the hearing loss, as expressed by the cochlear function as BC threshold. In case of conductive or mixed type of hearing loss with a $\mathrm{BC}$ threshold within $40 \mathrm{~dB}, \mathrm{BC}$ implants (BCIs) may be regarded as an easy and safe solution, which also assures that the preoperative hearing level does not deteriorate as a result of the surgical procedure. In contrast, when the $\mathrm{BC}$ threshold exceeds the $40 \mathrm{~dB}$ level, a BCI is less likely to produce a satisfactory hearing gain, especially when the contralateral ear is also hearing-impaired. Under these circumstances, the opportunity for the application of an AMEI, such as the Vibrant Soundbridge ${ }^{\circledR}$ oundbrarise.

After the initial application, which was reserved for pure sensorineural hearing loss with an intact ossicular chain, numerous reports on FMT application on the stapes, the footplate [12,13] or the RW [1] have shown fair auditory results. In respect to the simple procedure required for the application of a BCI, VSB surgery for a mixed type of hearing loss can be considered a demanding surgical procedure and, especially for the RW application, it requires to be performed by skillful and experienced surgeons if a good and stable functional outcome is to be achieved. The most important technical requisites can be individuated in: i) good contact of the FMT with the RWM, without bony contacts that may hamper or damp the vibratory mechanism, and ii) an inferior support for the FMT needed for an appropriate stabilization and tension of the device on the RWM [14]. In fact, to better exploit the electromechanical activity of the VSB FMT, and hence to obtain optimal stimulation of inner ear fluids, it is reasonable to assume that the upper side of the FMT should perfectly match the RW surface, without any bony contact or intermediate gap space. This issue is routinely checked intraoperatively by the experienced surgeon, and only rarely by applying objective reliable tests, mostly due to technical issues (ill-screen theatre, high impedance values, etc.). In this regard, the use of an imaging technique performed at a certain distance from surgery may be helpful to detect the detailed anatomic relationships between the FMT and the RWM.

In our series of RW-VP subjects, some degree of variability was found, such as to prompt us to carry out the present retrospective study, which aimed to individuate which factors may influence the relationship between the audiological outcome and the FMT/ RWM coupling as derived from the imaging, i.e. from the CBCT. In fact, contrary to the incus-VP, where FMT stabilization is assured by its firm crimping on the long incudal process, RW-VP encompasses some intraoperative uncertainty regarding the prediction of a successful outcome, because objective tests, such as electrocochleography, although suggested [10], are not routinely applied and represent a situation that may change after the completion of the healing period as well as in the long term. It is generally believed that the better the FMT/RWM coupling, the better the functional outcome.

In this retrospective study, the audiological and the radiological data were first independently assessed and then matched to evaluate whether the final auditory outcome was strictly related to a more or less appropriate placement of the FMT. CBCT equipment was selected for this purpose since, due to the 
Table III. Functional and radiological results in the individual cases of round window vibroplasty (RW-VP) at the low frequencies (125 and $250 \mathrm{~Hz})$.

\begin{tabular}{|c|c|c|c|c|c|c|}
\hline \multirow[b]{2}{*}{ Patient no. } & \multicolumn{3}{|c|}{ SF PTA2 $(125-250 \mathrm{~Hz})$} & \multicolumn{3}{|c|}{ CTCB parameters } \\
\hline & $\mathrm{U}$ & RW-VP & $\Delta$ & FMT/RWM contact & $\mathrm{BC}$ & IS \\
\hline 1 & 87.5 & 52.5 & +35 & ++ & Yes & No \\
\hline 2 & 77.5 & 67.5 & +10 & ++ & Yes & Yes \\
\hline 3 & 87.5 & 65 & +22.5 & +++ & No & No \\
\hline 4 & 87.5 & 55 & +32.5 & +++ & Yes & Yes \\
\hline 5 & 87.5 & 40 & +47.5 & ++ & Yes & No \\
\hline 6 & 82.5 & 75 & +5.5 & ++ & Yes & Yes \\
\hline 7 & 75 & 67.5 & +7.5 & ++ & Yes & No \\
\hline 8 & 45 & 45 & 0 & +++ & Yes & Yes \\
\hline
\end{tabular}

$\Delta$ : Difference value; BC: Bony contact; CTCB: Cone beam computed tomography; IS: Inferior support; PTA2: Pure-tone average in the 125 and $250 \mathrm{~Hz}$; SF: Sound field; U: Unaided; WRS: Word recognition score.

low radiation dosage in comparison with a conventional CT scan, it was considered to be a more ethical proposition for research purposes, particularly for those subjects who, due to optimal hearing results, would not have otherwise have undergone this procedure. Apart from the extremely low radiation dosage, CBCT is known also to provide excellent definition of the temporal bone, with very low scattering effect due to the metallic FMT [11]. A recent study has similarly reported on the use of this technique for analyzing specifically the situation after RWVP surgery, focusing on the distance and the angle of the FMT with respect to the RWM [15]. The same authors found some incongruence between an apparently well-positioned FMT and the auditory results. In the present study, we attempted to look in more detail at the FMT placement, taking into consideration three arbitrary parameters that should guarantee an optimal outcome, i.e. (a) correct FMT/RWM contact; (b) absence of bony contacts of the FMT on the RW niche; (c) appropriate inferior tissue support. It was possible to observe that the correct FMT/RWM contact (optimal or good) always led to a good functional outcome, even in the presence of bony contacts. It is also interesting that the postoperative hearing improvement that was not only shown, as expected, at the $500-4000 \mathrm{~Hz}$ range, but also at 125-250 frequency range, which is known not to be privileged by the technological features of the VSB (Table III). In this regard, it is possible to hypothesize that part of the beneficial effect could also be due to the amplification delivered by the bony contact of the FMT side on the promontory wall.

However, the present study has some limitations. First, a positive outcome was also found in subjects who showed some negative CBCT sign, such as the contact of the FMT to the bony lips of the RW, which would limit the importance of this parameter for the evaluation. On the other hand, the present radiological parameters were arbitrarily taken into consideration and presumably provide only a partial estimate of the FMT/ RWM coupling. Despite this consideration, it was possible to observe some useful correlation between these parameters and the audiological outcomes.

From these findings it is possible to conclude that CBCT can be considered a valid objective tool to assess in great detail the relationship between the FMT and the RW niche. Although such a diagnostic procedure may not be considered useful for those subjects who present satisfactory and long-standing auditory results, it may surely be helpful for detecting some local abnormalities in those subjects who show a hearing deterioration after a preliminary improved function, so as to be advised as a preoperative assessment before an eventual surgical revision.

Declaration of interest: The authors report no conflicts of interest. The authors alone are responsible for the content and writing of the paper.

\section{References}

[1] Colletti V, Soli SD, Carner M, Colletti L. Treatment of mixed hearing losses via implantation of a vibratory transducer on the round window. Int J Audiol 2006;45:600-8.

[2] Kiefer J, Arnold W, Staudenmaier R. Round window stimulation with an implantable hearing aid (Soundbridge) combined with autogenous reconstruction of the auricle - a new approach. ORL J Otorhinolaryngol Relat Spec 2006;68: 378-85.

[3] Luers JC, Hüttenbrink $\mathrm{KB}$, Zahnert $\mathrm{T}$, Bornitz $\mathrm{M}$, Beutner D. Vibroplasty for mixed and conductive hearing loss. Otol Neurotol 2013;34:1005-12.

[4] Linder T, Schlegel C, DeMin N, van der Westhuizen S. Active middle ear implants in patients undergoing subtotal 
petrosectomy: new application for the vibrant soundbridge device and its implication for lateral cranium base surgery. Otol Neurotol 2009;30:41-7.

[5] Beleites $T$, Neudert $M$, Beutner $D$, Hüttenbrink KB, Zahnert T. Experience with vibroplasty couplers at the stapes head and footplate. Otol Neurotol 2011;32: 1468-72.

[6] Zahnert T, Bornitz M, Hüttenbrink KB. Experiments on the coupling of an active middle ear implant to the stapes footplate. Adv Otorhinolaryngol 2010;69:32-7.

[7] Martin C, Deveze A, Richard C, Lefebvre PP, Decat M, Ibañez LG, et al. European results with totally implantable carina placed on the round window: 2-year follow-up. Otol Neurotol 2009;30:1196-203.

[8] Wever EG, Lawrence $M$. The functions of the round window. Ann Otol Rhinol Laryngol 1948;57:579-89.

[9] Monini S, Filippi C, Atturo F, Barbara M. Is the boneconduction head band test useful for predicting the functional outcome of a round window active middle ear implant? Otol Neurotol 2013;34:1329-35.
[10] Colletti V, Mandalá M, Colletti L. Electrocochleography in round window Vibrant Soundbridge implantation. Otolaryngol Head Neck Surg 2012;146:633-40.

[11] Güldner C, Heinrichs J, Weiß R, Eivazi B, Bien S, Teymoortash A, et al. Prospective evaluation of reliability of cone-beam computed tomography in detecting different position of vibroplasty middle ear implants. Clin Otolaryngol 2013;38:217-24.

[12] Beleites $T$, Neudert $M$, Beutner D, Hüttenbrink KB, Zahnert T. Experience with vibroplasty couplers at the stapes head and footplate. Otol Neurotol 2011;32:1468-72.

[13] Tsang WS, Yu JK, Wong TK, Tong MC. Vibrant Soundbridge system: application of the stapes coupling technique. J Laryngol Otol 2013;127:58-62.

[14] Salcher R, Schwab B, Lenarz T, Maier H. Round window stimulation with the floating mass transducer at constant pretension. Hear Res 2014;314:1-9.

[15] Edfelt L, Rask-Andersen H. Round window vibroplasty in chronic ear surgery. Comparison with conventional hearing rehabilitation. Acta Otolaryngol 2013;133:814-25. 